\title{
Geçmişten Günümüze Batarya Teknolojisi
}

\author{
Şükran Efe ${ }^{1}$, Zeynep Azra Güngör ${ }^{2 *}$ \\ ${ }^{1}$ Atatürk Üniversitesi, Mühendislik Fakültesi, Makine Mühendisliği Bölümü, Erzurum, Türkiye, (ORCID: 0000-0002-4033-6786), sukran.efe@atauni.edu.tr \\ ${ }^{2 *}$ Atatürk Üniversitesi, Fen Bilimleri Enstitüsü, Makine Mühendisliği Ana Bilim Dalı, Erzurum, Türkiye (ORCID: 0000-0001-6414-8395), \\ zeynepazra.gungor20@ogratauni.edu.tr
}

(International Conference on Design, Research and Development (RDCONF) 2021 - 15-18 December 2021)

(Uluslararası Araştırma-Geliştirme ve Tasarım Konferansı - 15-18 Aralık 2021)

(DOI: 10.31590/ejosat.1048673)

ATIF/REFERENCE: Efe, Ş. \& Güngör, Z. A. (2021). Geçmişten Günümüze Batarya Teknolojisi. Avrupa Bilim ve Teknoloji Dergisi, (32), 947-955.

\section{Öz}

İnsanoğlunun yaşamını idame edebilmesi için enerjiye olan bağımlılığı, her geçen gün artan enerji talebini oluşturmuş ve bunun sonucu olarak da dünya üzerinde en çok araştırma yapılan konuların başına enerjiyi taşımıştır. Günümüzde gelişen elektronik ve nano teknolojisinin ürünleri olan robotik, bilgisayar, cep telefonu, elektrikli araçlar, uzay sistemleri gibi teknolojik sistemler; yüksek enerji potansiyeline sahip, hafif, kolay ulaşılabilir, ucuz ve güvenilir enerji kaynağına ihtiyaç duymaktadır. Hareketli ve taşınabilir olan bu sistemlerde ihtiyaç duyulan enerjinin sürekliliği ve çevreyle dost olması kadar depolana bilirliği de önemli bir husustur. Günümüze kadar yürütülen enerji depolama çalışmalarında, depolanacak enerjinin çeşidine bağlı olarak; 1sıl enerjiyi, elektrik enerjisini, mekanik enerjiyi ve kimyasal enerjiyi depolama şeklinde birçok farklı metot denenmiştir. Bu çalışmada, teknolojik sistemlerde kullanımı yaygın bir şekilde tercih edilen, enerjinin kimyasal olarak depolandığı batarya teknolojisi ele alınmıştır. Çalışmada geçmişten günümüze kadar batarya çeşitleri, çalışma prensipleri, avantajları, dezavantajları karşılaştırmalı olarak incelenmiştir.

Anahtar Kelimeler: Bataryalar, Enerji Depolama, Karşılaştırma.

\section{Battery Technology From Past To Present}

\begin{abstract}
The dependence of human beings on energy for the survival of their lives has created an increasing demand for energy, and as a result, energy has become one of the most researched subjects in the world. Technological systems such as robotics, computers, mobile phones, electric vehicles, space systems, which are the products of developing electronics and nanotechnology; needs a light, easily accessible, cheap, and reliable energy source with high energy potential. In these mobile and portable systems, the continuity of the energy needed and its storability are as important as its environmental friendliness. In the energy storage studies carried out to date, many different methods have been tried in the form of storing thermal energy, electrical energy, mechanical energy, and chemical energy depending on the type of energy to be stored. In this study, battery technology, which is widely used in technological systems and where energy is stored chemically, is discussed. In the study, battery types, working principles, advantages, and disadvantages have been examined comparatively from past to present.
\end{abstract}

Keywords: Batteries, Energy Storage, Comparison

\footnotetext{
*Sorumlu Yazar: zeynepazra.gungor20@ogr.atauni.edu.tr
} 


\section{Giriş}

Gelişen teknoloji, artan dünya nüfusu ve konfor gereksinimlerinin etkisine, endüstriyel ve teknolojik alandaki yeniliklerde ilave edilince enerjinin tüketimi hızla artmaktadır. Enerji üretimi ile tüketimi arasındaki farkın her geçen gün arttığı günümüzde; enerji talebindeki hızlı artı̧ı karşılamak, alternatif enerji kaynaklarından kesintisiz yararlanabilmek, enerji sistemlerinin sürdürülebilir ve uzun ömürlü bir şseklide kullanabilmek ve en önemlisi de enerjinin verimliliğini artırmak amacıyla, enerjinin uygun bir şekilde depolanması gerekmektedir. Özellikle elektrikli otomobiller, akıllı telefonlar, fotoğraf makinaları, tabletler, bilgisayarlar, dronlar ve daha da yaygınlaşması beklenen IOT cihazların; yüksek enerji potansiyeline sahip, hafif, kolay ulaşılabilir, ucuz ve güvenilir enerji kaynağına ihtiyaç duyması, enerjinin depolana bilirliğinin önemini kat kat artırmıştır.

Doğanın bir parçası olan enerjiyi, birçok farklı metotla depolamak mümkündür. Isıl enerji depolama (katılarda depolama, sivilarda depolama, mevsimsel depolama, kimyasal depolama, faz değişimli maddelerle depolama), elektrik enerjisi depolama (ultra kapasitörler / süper kapasitörler, süper iletken manyetik enerji depolama, yakıt hücreleri), mekanik enerji depolama (hazneli pompalı sistemler, sıkıştırılmış hava ile enerji depolama, volanlar) ve kimyasal enerji depolama (piller, akış bataryaları) şeklinde enerji depolanabilmektedir (Kozak ve Kozak, 2012) (Yılmazoğlu, 2010). Son y1llardaki teknolojik gelişimlerin paralelinde, kontrol edilebilirliği ve taşınabilirliğinin daha iyi olması nedeniyle elektrokimyasal depolama, depolama teknikleri arasında belirgin bir şekilde ön plana çıkmaktadır.

Enerjinin kimyasal olarak depolanmasını sağlayan, bir veya daha fazla elektrokimyasal hücre, yakıt hücresi veya akış hücresi aracılı̆̆ıla kimyasal enerjiyi elektrik enerjisine dönüştüren depolama birimlerine pil denir. Pillerin bir araya gelerek oluşturdukları pil grupları "batarya" olarak tanımlanmaktadır (Winter and Brodd, 2004) (Güven ve Gedik, 2019). En eski insan yapısı pillerin M.Ö. 250 ve M.S. 640 yılları arasında kullanılan Bağdat Pilleri olduğu bilinmektedir (Von Handorf, 2002). İtalyan fizikçi Alessandro Volta'nın adının da verildiği Volta pili ya da Kaplar Zinciri 1800 yılında bulunan ilk pildir. Pil tasarımı 1836 yllında John Frederick Daniell tarafindan standart haline getirilmiş ancak ticari olarak kullanımı 1991'de Sony ve Asashi Kasei tarafindan gerçekleştirilmiştir (Kul, 2020).

250 yıllık tarihsel gelişimine rağmen genel yapısında değişiklik olmayan pillerdeki enerji üretimi; biri pozitif diğeri negatif iki elektrot arasına yerleştirilen elektrolitte içindeki indirgenme-yükseltgenme (redoks) reaksiyonlarından ibarettir. Günümüze kadar elektrotlarda ve/veya elektrolitte değişiklikler yapılarak birçok farklı pil araştırma çalışması yürütülmüş olup yeni araştırma çalışmaları devam etmektedir.

$\mathrm{Bu}$ çalışmada amaç, günümüz de depolanabilir enerjinin vazgeçilmezi olan bataryaların geçmişten bugüne teknolojik gelişimini incelemektir. Bu bağlamda; bataryaların çalışma prensibi, yaygın kullanılan çeşitleri, avantajları/dezavantajları literatürdeki güncel çalışmalar ele alınarak detaylı bir şekilde incelenmiş ve karşılaştırmalı olarak sunulmuştur.

\section{Materyal ve Metot}

Cep telefonu, radyo, dizüstü bilgisayar vb. elektronik cihazlardan, elektrikli araçlara, uydu ve uzay sistemlerinden, haberleşme sistemleri ve savunma sistemlerine kadar oldukça geniş bir kullanım alanına sahip olan bataryalar elektrik enerjisini kimyasal enerji olarak depolayan birimlerdir. Bataryalar depo ettikleri kimyasal enerjiyi, elektrokimyasal redoks reaksiyonları aracıllğıyla elektrik enerjisine dönüştürme prensibine göre çalışmaktadır. Redoks reaksiyonları bir elektrik devresi aracılığıla elektronların bir maddeden diğerine aktarılmasıdır. Batarya sistemi, istenilen çıkış gerilimini ve kapasiteyi elde edebilmek için seri, paralel veya hem seri hem de paralel kompleks bir şekilde bağlanmış, bir veya daha çok hücreden meydana gelmektedir (Çetin vd., 2021). Her bir hücre, metal anot (negatif elektrot), metal oksit katot (pozitif elektrot) ile bu iki elektrot arasında kimyasal reaksiyonu sağlayan elektrolitten ibarettir [Şekil 1]. Anot, elektrolizde aşınırken katotta iyonik değişim reaksiyonu sonucu elektrik akımı meydana gelir. $\mathrm{Bu}$ reaksiyon sonucu oluşan elektrik enerjisi çeşitli aletlerde kullanılır (Dinçer ve Ezan, 2020)

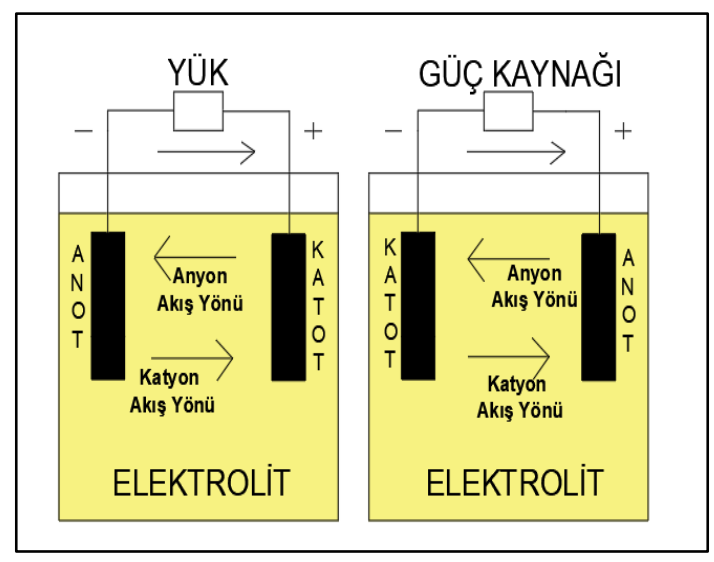

Şekil 1. Pilin Yapısı

Bataryaların karakteristik özellikleri, bataryalarda kullanılan hücrelerin türü, hücrelerin sayısı, hücrelerin seri-paralel bağlantı yapısı ve bataryalarda kullanılan hücrelerin dışındaki donanımsal elemanlar tarafindan belirlenir. Bataryalar, şarj edilebilir bataryalar ve şarj edilemeyen bataryalar olarak ikiye ayrilırlar. Şarj edilemeyen bataryalar "birincil bataryalar" olarak isimlendirilirken, şarj edilebilen bataryalar ise "ikincil bataryalar" olarak isimlendirilirler. Şarj edilebilir yani ikincil bataryalar, deşarj olduktan sonra tekrar tam şarj edilerek kullanılabilen batarya türleridir. Aynı zamanda depolama bataryaları ve akümülatörler olarak da adlandırılırlar. İkincil bataryalar, yüksek güç yoğunluğu, yüksek deşarj oranı, düz deşarj eğrileri ve uygun düşük sıcaklık performansı ile karakterize edilirler (Çetin vd., 2021).

Günümüze kadar gelen batarya çalışmalarında, bataryalar kullanılan elektrot ve/veya elektrolit çeşidine göre; kurşun-asit, nikel-kadmiyum, nikel-metal hidrit, lityum-iyon ve lityum-sülfür bataryalar olarak sinıflandırılmaktadır.

\subsection{Kurşun-Asit (Pb-Asit) Bataryalar}

Kurşun elektronik cihazlarda yaygın olarak kullanılmaktadır. Örneğin; kurşun, katot 1şını tüplerinin (televizyonlar ve monitörler) camında lehimlerin ve kurşun oksitlerin ana bileşeni olarak kullanılabilirken, kurşun asit bataryalarda ve bazı PVC kablolarda stabilizatör olarak kullanılabilir (Zheng, et al., 2008) (Zhang Q. , 2013). Pb-asit bataryalar birçok uygulamada kullanılmakta olan eski ve yaygın bir teknolojidir. Fransız fizikçi Gaston Plante tarafindan 1859 yllında icat edilen ilk ticari ve şarj edilebilir pil türüdür (Kurzweil, 2010) (Calborean, et al., 2021). 
Pb-asit bataryalar günümüzde otomobil, ulaşım ve telekomünikasyon endüstrisinde en çok kullanılan batarya çeşididir (Zhang Q. , 2013).

$\mathrm{Pb}$-asit bataryalar da anotta $\mathrm{Pb}$ ve katotta ise $\mathrm{PbO}_{2}$ yer almaktadır [Şekil 2]. Kurşun metalden yapılmış negatif bir plakaya ve her ikisi de seyreltilmiş kükürt asidinden oluşan bir elektrolit içine batırılmış kahverengi $\mathrm{PbO}_{2}$ kurşun dioksitten pozitif bir plakaya sahiptir. Elektrik enerjisi kurşun-asit aküde depolanır ve kimyasal enerjiden elektrik enerjisine dönüştürülebilir. Piller elektrokimyasal reaksiyonlarla enerji depolar ve boşaltır. Deşarj aşamasında, her iki elektrot da kurşun sülfata $\left(\mathrm{PbSO}_{4}\right)$ dönüşecek ve elektrolit çözeltisinin sülfat iyonlarını tüketecektir (Islam, et al., 2021). İlk yükleme işlemi sırasında, $\mathrm{PbSO}_{4}$ negatif plakalarda metale indirgenirken pozitiflerde kurşun oksit oluşur $\left(\mathrm{PbO}_{2}\right)$ (Sanguesa, et al., 2021). Deşarj sırasında elektronlar yükten dışarıdan geçirilirken, elektrolit ve elektrotların aralığındaki iç kimyasal reaksiyonlar yük dengesini dengelemek için çalışır.

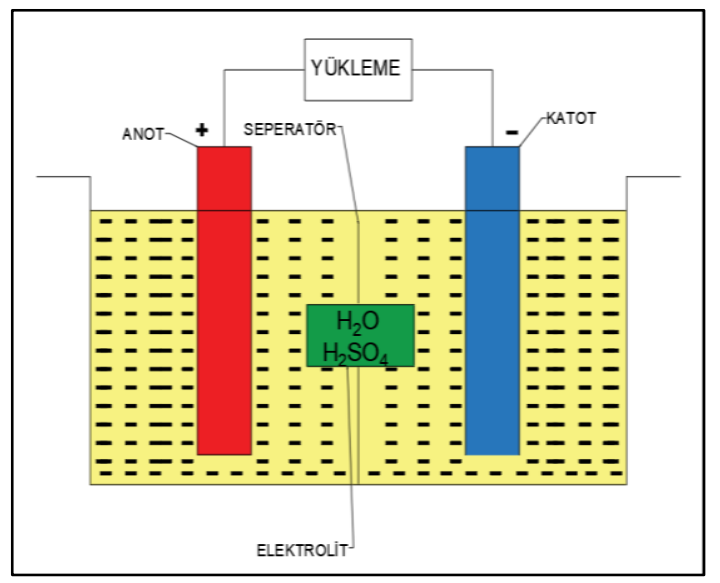

Şekil 2. Kurşun-asit pil şeması

Pb-asit bataryalar; güçlü geri kazanım yeteneği, düşük maliyet, mükemmel güvenlik performansı, kullanım aşamasında uzun bir yaşam döngüsü, düşük kendi kendine deşarj oranı, düşük bakım (genelde bakım gerektirmeme), çeşitli boyut ve tasarımlara sahip büyük miktarlarda kullanılabilirlik, geniş bir sicaklık aralığında etkili bir şekilde çalışabilme yeteneği gibi özelliklere sahiptir (Lach, et al., 2019) (Jung, et al., 2021) (Dong, et al., 2021). Bu bataryaların dezavantajları arasında sınırlı enerji yoğunluğu, nispeten düşük çevrim ömrü, uzun şarj süresi, çevre kirliliği, yüksek kütle, termal dengesizlik, kalıcı kapasite kaybı gibi problemler yer almaktadır. Depolamada kendi kendine deşarj hızları yüksektir. Aşırı şarj işlemi elektrotların geri dönüşü olmayan polarizasyona ve deşarjda iken uzun süreli depolamada sülfat üretimine neden olmaktadır. (Nazghelichi et al., 2021) (Witantyo, et al., 2021) (Bulut and Özcan , 2021) (Samanta and Chowdhuri, 2021)

$\mathrm{Pb}$-asit bataryaların nominal gerilimi ve enerji yoğunlukları diğer batarya teknolojilerine göre düşüktür. Lityum iyon bataryaların gerilim değerinin yüksek olması kurşun-asit pilleri tercih konusunda öteye atmaktadır. Kurşun-asit bataryanın $2 \mathrm{~V}$ gerilim değerine sahipken lityum iyon bataryaları ile karşılaştırıldığında yetersiz kalmakta ve bu gerilim değerine ulaşabilmek için daha fazla hücrenin kullanımı nedeniyle son yıllarda üstün özelliklere sahip piller daha fazla tercih konusu olmaktadır.

\subsection{Nikel-Kadminyum (Ni-Cd) Bataryalar}

Nikel (Ni), korozyona ve termal bozulmaya karşı oldukça yüksek direnç gösteren gümüşi beyaz, güçlü ve sünek bir metaldir (Aruna, et al., 2013). Korozyona karşı koruma sağlamak için diğer metallerin kaplanmasında yaygın olarak kullanılır ve tokluğu, çekme mukavemetini ve elastik limiti artırmaya sağladığı için metal alaşımlarının üretiminde yaygın olarak kullanılır (Roth, et al., 1997).

Ni-Cd batarya sistemi ilk olarak 1899 yılında İsveçli Waldemar Jungner tarafından yapılmış (Ba"uerlein, et al., 2008), üretimi 1950 yılında Avrupa ve Amerika Birleşik Devletleri'nde ortaya çıkmıştır (Saleh, et al., 2021). Yangın alarm sistemleri, sinyalizasyon ve telekomünikasyon, güneş fotovoltaik, enstrümantasyon ve proses kontrolü, şalt koruması, acil aydınlatma, ups, elektromanyetik kaldırıcılar (eot vinç) gibi farklı uygulamalar için kullanılır (Kotkunde, et al., 2021).

Ni-Cd bataryalarda, anotta kadmiyum metali ve katotta ise $\mathrm{Ni}(\mathrm{OH}) 2$ içermektedir [Şekil 3]. Pozitif elektrottan salınan elektronlar kadmiyum ve hidroksit ile negatif elektrota gider ve kadmiyum hidroksit oluşur. Elektroliti potasyum hidroksittir $[\mathrm{KOH}]$. Reaksiyon, tersinir olduğu için batarya şarj edilebilir.

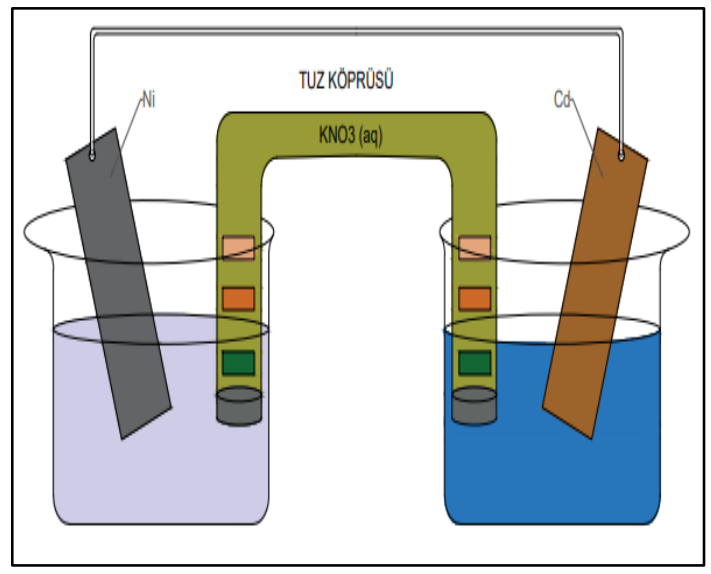

Şekil 3. Ni-Cd pil şemast

Ni-Cd çifti; yüksek enerji kapasitesine, uzun çalışma ömrüne, yüksek güvenilirliğe, daha hızlı şarj ve yüksek deşarj hızları gerektiren uygulamalarda kullanılır (International Energy Agency, 2013). Ayrıca, geniş bir çalışma sıcaklık aralığı, neredeyse tamamen geri dönüştürülebilir, bakım gerektirmeyen, mekanik ve elektriksel suistimallere karşı yüksek direnç gösterebilme gibi avantajlara sahiptir (Putois, 1995). Yapısındaki ağır metaller nedeniyle Ni-Cd bataryaların yaygın kullanımı çevre kirliliğini artırarak çevreye zarar verir. $\mathrm{Bu}$ nedenle kullanımı sınırlandırlmıştır. Bataryanın yapısındaki kalıntılar, küçük kadmiyum blokları oluşturarak hafıza etkisi adı verilen ve batarya performansını önemli derecede etkileyen bir soruna neden olmaktadır. Kendi kendine deşarj (aylık \%20) özelliği yüksektir. Cd elementinin yüksek maliyeti nedeniyle pahalı bir eneri depolama sistemidir (Martinez-Bolanos, et al., 2020) (Nandiwale, 2021). Diğer batarya türleri ile karşılaştırıldığın da enerji yoğunluğu düşüktür. Özellikle çevreye olumsuz etkilerinin bertaraf edilmesi amaciyla Cd kullanılmayan nikel metal hidrit bataryaların oluşumuna neden olmuştur.

\subsection{Nikel-Metal Hidrit (Ni-MH) Bataryalar}

$\mathrm{Ni}-\mathrm{MH}$ bataryalar, en iyi batarya teknolojilerinden biri olup, Stanford R. Ovshinsky tarafından patenti alınmış ve 1982 yılında Ovonic Battery şirketi tarafından kurulmuş (Ba"uerlein, et al., 
2008) ve 1990'lı yıllarda ticarileştirilmiştir (Muthu and Sinnaeruvadi , 2021). Kameralar, dizüstü bilgisayarlar, tıbbi cihazlar ve sayısız diğer taşınabilir elektronik cihazlar dahil olmak üzere birçok cihazda yaygın olarak uygulanan ve Ni içeren bataryaların ana türüdür. Elektrikli araçlarda ve hibrit elektrikli araçlarda da kullanılmaktadır (Odegbemi, et al., 2001).

Ni-MH bataryalar, anotta hidrojen adsorblama özelliği olan metal hidrür ve katotta ise $\mathrm{Ni}-\mathrm{Cd}$ pillerde ki gibi $\mathrm{Ni}(\mathrm{OH})_{2}$ içermektedir [Şekil 4]. Kullanılan elektrolit bir potasyum çözeltisidir. İki elektrot arasında ayırıcı olarak genellikle poliamid veya polipropilen keçe veya gazlı bez kullanılır. Batarya çelik bir kasaya yerleştirilmiştir. Silindirik hücrelerde anot, katot ve ayırıcı şeritler lamine edilir ve spiral şeklinde sarılır. Elektrolit hücrenin merkezine doldurulur ve ardından hücre kapatılır (Müller and Friedrich, 2006). Ni-MH bataryaların elektrokimyasal performansı, hidrojeni tersinir bir şekilde depolayabilen MH negatif elektrot malzemesine güçlü bir şekilde bağlıdır (Ouyang, et al., 2017). Batarya deşarj edildiğinde, negatif elektrottaki metal hidrit, metal alaşım oluşturmak için oksitlendirilir ve pozitif elektrottaki $\mathrm{Ni}(\mathrm{OH})_{2}$ meydana getirilir. Şarj sırasında ters reaksiyon meydana gelir ve enerji depolanır (Nitesh and Ravichandra, 2021)

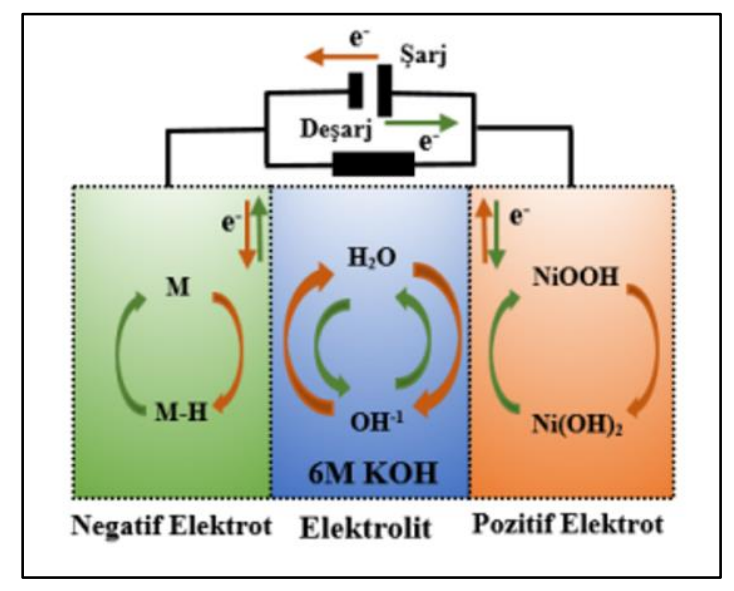

Şekil 4. Ni-MH Pil Şeması (Küçükdeveci, 2018)

Ni-MH bataryalar; mükemmel güvenliği, düşük maliyeti, uzun çevrim ömrü, çevre dostu, düşük sıcaklıklarda yüksek deşarj kapasitesi, aşırı şarj/deşarj toleransı, iyi aktivasyon kabiliyeti, toksik olmama ve yüksek güç çıkışı gibi temel özellikleri nedeniyle ideal bir enerji kaynağı olarak kabul edilmiştir (Moller, et al., 2017) (Ouyang, et al., 2017) (Chen, et al., 2021). Bu bataryaların en büyük sınırlaması, diğer ikincil hücrelere kıyasla yüksek çalışma sıcaklığında yüksek kendi kendine deşarj hızı, düşük güç ve enerji yoğunluğuna sahip olmsıdır. Bir diğer dezavantajı da kapasite tutma etkisinin olmamasidır (Silvestri, et al., 2020) (Wang, et al., 2021).

$\mathrm{Pb}$-asit ve Ni-Cd bataryalar ile kıyaslandığında, $\mathrm{Pb}$ ve $\mathrm{Cd}$ içermediğinden, çevreyle daha dost bir bataryadır. Maliyet bakımından da lityum-iyon bataryalara kıyasla daha uygundur.

\subsection{Lityum-Iyon (Li-iyon) Bataryalar}

Lityum metali, genel olarak şarjedilebilir bataryalarda (cep telefonu, dizüstü bilgisayarlar, tabletler vb.) ve ağırla yüksek direniş göstermesi sebebiyle alaşım olarak hava taşıtlarında kullanılmaktadır (Çifçi and Meriç, 2021). Lityum içerikli bataryalar, hafif olmasıyla ön plana çıkmaktadır (Gül, vd., 2012). Li-iyon bataryalar, ilk olarak 1991 yılında Sony tarafindan ticarileştirilmiştir (Bae and Kim, 2021). Günümüzde taşınabilir elektronik cihazlarda (cep telefonları, dizüstü bilgisayarlar ve tabletler), elektrikli araçlarda ve tıbbi cihazlardaki özellikle yüksek çevrim sayısı nedeniyle yaygın olarak kullanılmaktadır (Chan, et al., 2008) (Kumar, et al., 2019).

Li-iyon batarya, organik sıvı elektrolit içine batırılmış ve gözenekli ayırıcı ile ayrılmış katot ve anot elektrotlarından oluşmaktadır [Şekil 5]. Lityum atomu şarj esnasında elektron vererek $\mathrm{Li}^{+}$katyonunu ouşturur ve katottan sulu olmayan elektrolit içine çözülür, elektrolit/ayırıcı aracılığıyla katottan anot tarafina çaprazlanır ( $\mathrm{Xu}$, et al., 2013). $\mathrm{Li}^{+}$iyonları reaksiyona doğrudan girmez, anot/katot yapısına yerleşir ve ayrılır (Xu, et al., 2012).

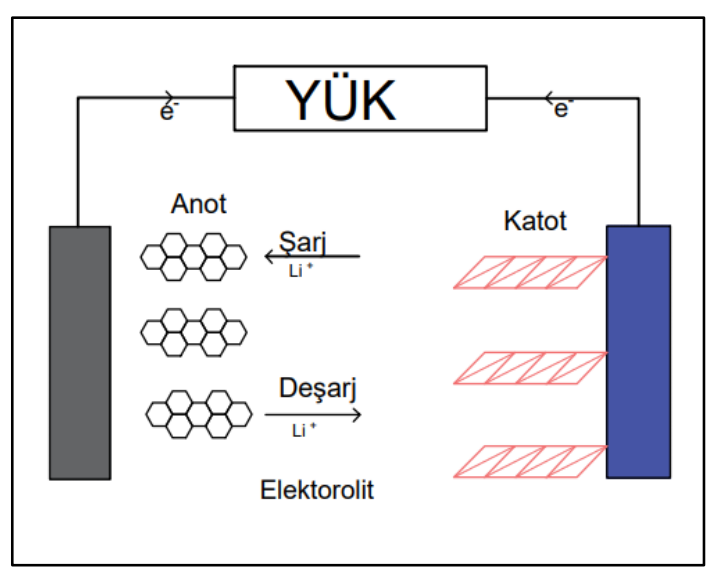

Şekil 5. Lityum-iyon Pil Şeması

Lityum-iyon bataryaların; yüksek enerji ve güç yoğunluğu, düşük maliyet, düşük kendi kendine deşarj, düşük çevre kirliliği, geri döndürülebilir ve uzun çalışma süresi, yüksek güvenilirlik, uzun çevrim ömrü, daha hızlı şarj/deşarj oranları gibi üstün özelliklere sahiptir (You, et al., 2021) (Lu, et al., 2021) (Zhou, et al., 2021) (Lin, et al., 2021) (Lyu, et al., 2021). Sinırlı kapasite, şarj/deşarj esnasında eletrotlarda hacimsel artıştan kaynaklanan patlama riski ise en belirgin dezavantajıdır. (Makuza, et al., 2021) (Chen, et al., 2021).

\subsection{Lityum-Sülfür (Li/S) Piller}

Lityum katı hâldeyken en hafif metallerden biri olup $(6,94$ $\mathrm{g} / \mathrm{mol}$ ) değerine sahiptir (Xie and Lu, 2020). Lityumun teorik spesifik kapasitesi 3860 mAhg $^{-1}$ dır (Wang, et al., 2011). Sülfür ise doğada en çok bulunan elementler arasında yer almaktadır (Bucur, et al., 2017). Sülfür bilinen katotlardan en yüksek teorik kapasiteye ve en yüksek teorik güç yoğunluğuna sahip bir bileşendir (Nagao, et al., 2011). S' ün teorik spesifik kapasitesi

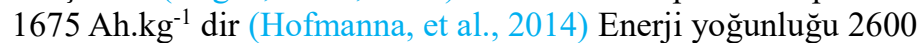
Wh.kg ${ }^{-1}$ dır (He, et al., 2011) Sülfür ekonomik açıdan ucuz bir elementtir (Cai, et al., 2012). S'ün diğer bir özelliği de çevre ile dost bir enerji kaynağı olmasıdır (Conder, et al., 2017). Elektrik iletkenliği düşük ve $1 \times 10^{-15} \mathrm{Sm}^{-1}$ dir (Ding, et al., 2014). Yalıtkanlık değeri $5 \times 10^{-30} \mathrm{Scm}^{1}{ }^{\prime} \mathrm{dir}$ (Ye, et al., 2016).

Bataryalarda S'ün katot materyali olarak kullanımı ilk olarak 1962 yılında Ulam ve Herbert tarafından yapılmış ve 1967 yılında Argon Ulusal Laboratuvarında yürütülmeye başlatılmıştır (Yuan, et al., 2013) (Sylwia, 2015). Pilin yapısı çok karmaşık olmamak ile birlikte bir kasa içerisine sıkıştırılmış anot, katot, elektrolit ve ayırıcılardan oluşmaktadır [Şekil 6]. 


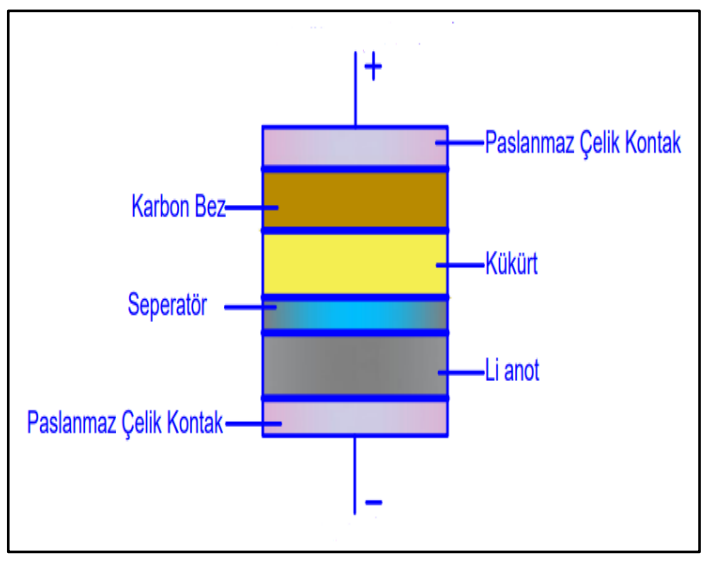

Şekil 6. Lityum-sülfür batarya şeması

$\mathrm{Li} / \mathrm{S}$ bataryalarda anotta Li metali, katotta elementel S $\left(\mathrm{S}_{8}\right)$ toz halde bulunur. Çalışma mekanizması, katı-çözeltikatı geçişini içeren $\mathrm{Li}$ ve $\mathrm{S}$ arasındaki karmaşık çok aşamalı redoks reaksiyonlarına dayanmaktadır (Chen, et al., 2015) . Şarj işlemi; $\mathrm{S}_{8}$ 'nin lityum ile reaksiyonu sonunda lityum sülfit $\left(\mathrm{Li}_{2} \mathrm{~S}\right)$ olarak tamamen indirgenme reaksiyona dayanır (Zhang and J.A., 2012). S8, her atomu için iki adet Li ile reaksiyona girdiği için geleneksel pozitif aktif elektrot malzemelerden çok daha yüksek teorik kapasiteye sahiptir (Tanibata, et al., 2017) (Ding, et al., 2014). Deşarj sırasında $\mathrm{S}_{8}$ 'in halka yapısı açılır ve farklı zincir uzunlunlarında, poly-sülfit (PS) olarak isimlendirilen yapısına dönüşür [Şekil 7] (Walus S., 2015) (Zhang S., 2013) (Azimi, et al., 2015 ). Deşarj sırasında, pozitif elektrot üzerinde üretilen çözünür PS'ler, redoks olarak bilinen bir işlemle negatif elektrot (redüksiyon) ve pozitif elektrot (oksidasyon) arasında mekik dokur (Conder, et al., 2017). Şarj işleminde çözünmeyen PS, çözünür PS'ye oksitlenir ve bu da daha fazla sülfür haline getirilir ve şarj tamamlandığında karbon yüzeyine çökeltir. Elektrolitteki fazla PS miktarı, sülfür yüzeyini iletken olmayan bir kaplama olarak kaplayabilir ve deşarj performansını olumsuz yönde etkileyebilir (Ding, et al., 2014) (Nagata and Chikusa, 2016) Birçok araştırmacı, şarj sırasında $\mathrm{Li}_{2} \mathrm{~S}_{\mathrm{X}}$ çözeltisini önlemek için sülfür malzemelerini mezoporöz karbona hapsetmeye odaklanmıştır. Sülfür yüksek dereceli mezoporöz karbona dahil edilerek, ardından polietilen glikol ile kaplanarak (Ji, et al., 2009) (Ding, et al., 2014) veya karbon siyahının yerine grafen ya da karbon nanotüp yükü kullanılarak kullanılabilir (Zhao, et al., 2012) (Jin, et al., 2013). Grafen, Li/S hücresi için uygulanabilir bir karbon matrisi malzemesidir, çünkü son derece iletkendir ve PS çözünmesini engellemek için sülfürü kaplar (Evers and Nazar, 2012).

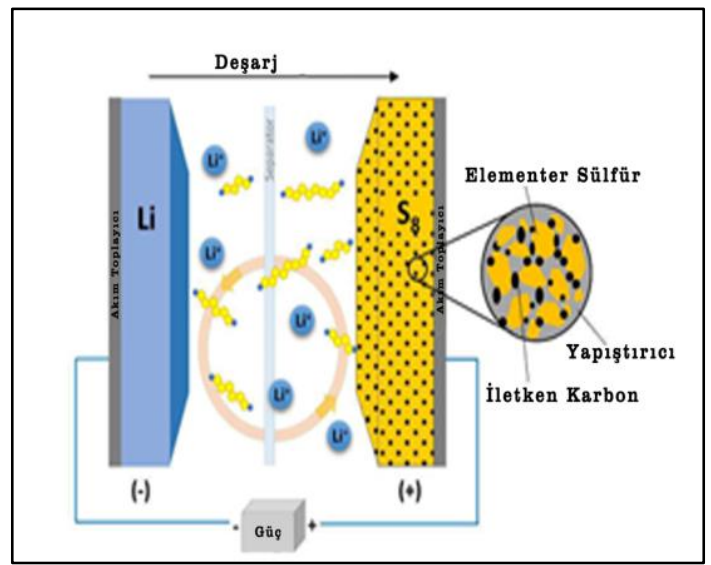

Şekil 7. Li/S batarya şeması

Li/S bataryalar, yeni nesil batarya sınıfnda, yüksek enerji potansiyeline sahip (Li-iyon bataryalardan 3,5 kat daha yüksek), diğer bataryalara göre hafif, ani ısı değişimine, geniş bir çalışma sıcaklığı aralığına, ezilmelere gibi bir çok olumsuz etkiden uzaktır. Depolama kapasiteleri yüksek, çevreyle dost, hiçbir bakım gerektirmeyen, ve raf ömürleri oldukça uzundur. Sülfürün doğada bol miktarda bulunması ve ucuz olması, bataryayı da düşük maliyetli bir sistem haline getirmektedir (Cleaver, et al., 2018) (Wu, et al., 2018) (Xu, et al., 2019) Diğer taraftan; sınırlı çevrim ömrü, düşük şarj verimi, zayıf güvenlik, yüksek kendi kendine deşarj oranı gibi dezavantajlarına sahiptir (Ma, et al., 2015) (Liu and Cui, 2017) (Stroe, et al., 2019)

\section{Araştırma Bulguları}

Geçmişten günümüze kadar batarya teknolojilerindeki durumun incelendiği bu çalışmada; bataryaların çalışma prensipleri, avantajları, dezavantajları tanımlanmıştır. Bataryaların teknik özellikleri karşılaştırılmıtır [Tablo 1]. 


\begin{tabular}{|c|c|c|c|c|c|c|c|c|}
\hline $\begin{array}{c}\text { Pil } \\
\text { Türü }\end{array}$ & 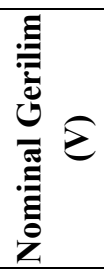 & 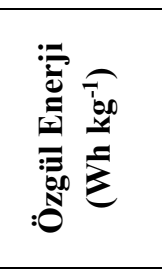 & 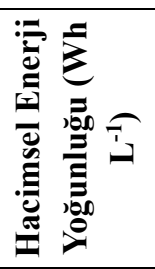 & 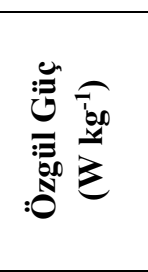 & 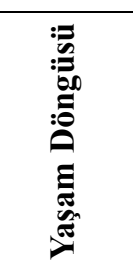 & 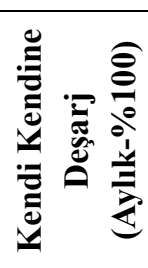 & 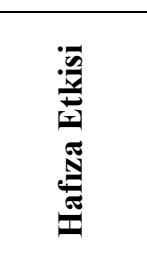 & 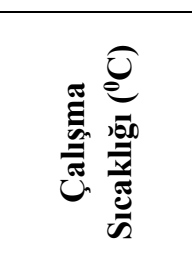 \\
\hline Pb-Asit & $2,0^{\mathrm{a}, \mathrm{b}}$ & $35^{\mathrm{a}, \mathrm{b}}$ & $100^{\mathrm{a}}$ & $180^{\mathrm{a}}$ & $1000^{\mathrm{a}, \mathrm{b}}$ & $<5^{\mathrm{a}}$ & Hayır ${ }^{\mathrm{a}, \mathrm{b}}$ & -15 ile $+50^{\mathrm{a}, \mathrm{b}}$ \\
\hline $\mathrm{Ni}-\mathrm{Cd}$ & $1,2^{\mathrm{a}}$ & $50-80^{\mathrm{a}, \mathrm{b}}$ & $300^{\mathrm{a}}$ & $200^{\mathrm{a}}$ & $2000^{\mathrm{a}}$ & $10^{\mathrm{a}}$ & Evet $^{\mathrm{a}, \mathrm{b}}$ & -20 ile $+50^{a, b}$ \\
\hline Ni-MH & $1,2^{\mathrm{a}, \mathrm{b}}$ & $70-95^{\mathrm{a}, \mathrm{b}}$ & $180-250^{\mathrm{b}}$ & $200-300^{\mathrm{a}}$ & $<3000^{a, b}$ & $20^{\mathrm{a}}$ & Seyrek $^{\mathrm{a}, \mathrm{b}}$ & -20 ile $+60^{a, b}$ \\
\hline Li-iyon & $3,6^{\mathrm{a}, \mathrm{b}}$ & $118-250^{\mathrm{a}, \mathrm{b}}$ & $200-400^{\mathrm{a}}$ & $200-430^{\mathrm{a}}$ & $2000^{\mathrm{a}, \mathrm{b}}$ & $<5^{\mathrm{a}}$ & Hayır ${ }^{\mathrm{a}, \mathrm{b}}$ & -20 ile $+60^{a, b}$ \\
\hline Li-S & $2,5^{\mathrm{a}}$ & $350-650^{\mathrm{a}, \mathrm{b}}$ & $350^{\mathrm{a}}$ & - & $300^{\mathrm{a}}$ & $8-15^{\mathrm{a}}$ & Hayır & -60 ile $+60^{a, b}$ \\
\hline
\end{tabular}

a Ref (Özcan, vd., 2021)

b Ref (Muratoğlu ve Alkaya, 2016)

\section{Sonuç}

Çevre kirliliğin azaltılması, enerjide dışa bağımlılığının düşürülmesi ve enerji maliyetinin minimilize edilmesi, arz güvenliğinin sağlanması için enerji depolamanın önemi her geçen gün arttığ1 günümüz de, teknolojik gelişmeler ile birlikte de batarya teknolojisinin de önemi giderek artmaktadır. Son yıllarda batarya teknolojisi gelecek vadeden yaygın olarak çalışılan konular içerisindedir. $\mathrm{Bu}$ çalışma kapsamında bataryaların dünden bugüne olan gelişimi incelemek amacıyla batarya çeşitleri (kurşun-asit, nikel-kadminyum, nikel-metal hidrit, lityum-iyon ve lityum-sülfür) incelenmiştir. Her bir batarya çeşidinin çalışma prensibi, avantajı, dezavantajı hakkında bilgi sunulan çalışmada, bataryaların teknik özellikleri de karşılatırılmalı olarak sunumuştur. Yapılan araştırma çalışmasıyla; çalışmada lityumiyon bataryalarının diğer batarya çeşitlerine göre nomina gerilim ve özgül güç değerlerinin daha büyük olduğu, sadece nikelkadminyum bataryaların da hafıza etkisinin görüldüğü, diğer bataryalara göre nikel-metal hidrit bataryalarının da daha uzun bir yaşam döngüsüne sahip olduğu görülmektedir. Batarya çeşitleri içerisinde lityum-sülfür bataryalarının özgül enerji, hacimsel enerji yoğunluğu kendi kendine deşarj ve çalışma sıcaklığının daha iyi değerlere sahip olduğu görülmektedir. Bu çalışma günümüz bataryalarına genel bir bakış yapan derleme çalışamsı niteliğindedir.

\section{Kaynakça}

Aruna, S. T., Savitha, G., Shedthi, J., \& William Grips, V. K. (2013). The Corrosion Resistance of Nickel Electrocomposite Coating Containing BaFe12O19 Particles. ISRN Corrosion, 6 pages. doi:http://dx.doi.org/10.1155/2013/192684

Azimi, N., Xue, Z., Hu, L., Takoudis, C., Zhang, S., \& Zhang, Z. (2015). Additive Effect on the Electrochemical Performance of Lithium-Sulfur Battery. Electrochimica Acta.(154), 205210. doi:https://doi.org/10.1016/j.electacta.2014.12.041

Bäuerlein , P., Antonius, C., Löffler, J., \& Kümpers, J. (2008). Progress in high-power nickel-metal hydride batteries. Journal of Power Sources, 176, 547-554. doi:http://dx.doi.org/10.1016\%2Fj.jpowsour.2007.08.052

e-ISSN: 2148-2683
Bae , H., \& Kim, Y. (2021). Technologies of lithium recycling from waste lithium ion batteries: a review. Mater. Adv., 2, 3234-3250. doi:https://doi.org/10.1039/D1MA00216C

Bucur, C., Jones, M., Kopylov, M., Spearb, J., \& Muldoon, J. (2017). Inorganic-organic layer by layer hybrid membranes for lithium-sulfur batteries. Energy \& Environmental Science(10), 905-911. doi:https://doi.org/10.1039/C7EE00398F

Bulut , M., \& Özcan , E. (2021). A novel approach towards evaluation of joint technology performances of battery energy storage system in a fuzzy environment. Journal of Energy Storage,

36(102361). doi:https://doi.org/10.1016/j.est.2021.102361

Cai, K., Song, M., Cairns, E., \& Zhang, Y. (2012). Nanostructured Li2S-C Composites as Cathode Material for HighEnergy Lithium/Sulfur Batteries. Chem. Soc.(12), 6474-6479. doi:https://doi.org/10.1021/n1303965a

Calborean, A., Murariu, T., \& Morari, C. (2021). Optimized leadacid grid architectures for automotive lead-acid batteries: An electrochemical analysis. Electrochimica Acta, 372(137880). doi:https://doi.org/10.1016/j.electacta.2021.137880

Chan, C. K., Peng, H., Liu, G., Mcilwrath, K., Zhang, X. F., Huggins, R. A., \& Cui, Y. (2008). High-performance lithium battery anodes using silicon nanowires. nature nanotechnology, 31-35. doi:https://doi.org/10.1038/nnano.2007.411

Chen, H., Wang, C., Dong, W., Lu, W., Du, Z., \& Chen, L. (2015). Monodispersed Sulfur Nanoparticles for Lithium-Sulfur Batteries with Theoretical Performance. Chem. Soc(15), 798802. doi:https://doi.org/10.1021/nl504963e

Chen, M., Tan, C., Jiang, W., Huang, J., Min, D., Liao , C., . . . Zhu, M. (2021). Influence of over-stoichiometry on hydrogen storage and electrochemical properties of Sm-doped low-Co AB5-type alloys as negative electrode materials in nickelmetal hydride batteries. Journal of Alloys and Compounds, 867(159111). doi:https://doi.org/10.1016/j.jallcom.2021.159111

Chen, Z., Shi, N., Ji, Y., Niu, M., \& Wang, Y. (2021). Lithiumion batteries remaining useful life prediction based on BLSRVM. Energy, 234(121269), 13 pages. doi:https://doi.org/10.1016/j.energy.2021.121269 
Clean Energy Ministerial, Electric Vehicles İnitiative, International Energy Agency. (2013). Understanding the electric vehicle landscape to 2020. International Energy Agency. Paris: IEA, Global EV Outlook.

Cleaver, T., Kovacik, P., Marinescu, M., Zhang, T., \& Offer, G. (2018). Commercializing Lithium Sulfur Batteries: Are We Doing the Right Research? Journal of The Electrochemical Society., 1(165), A6029-A6033. doi:http://dx.doi.org/10.1149/2.0071801jes

Conder, J., Bouchet, R., Trabesinger, S., Marino, C., Gubler, L., \& Villevieille, C. (2017). Direct observation of lithium polysulfides in lithium-sulfur batteries using operando X-ray diffraction. Nature Energy(2), 1-7. doi:http://dx.doi.org/10.1038/nenergy.2017.69

Çetin, M. S., Karakaya, B., \& Gençoğlu, M. T. (2021). Elektrikli Araçlar İçin Lityum İyon Bataryaların Modellenmesi. Fırat Üniversitesi Müh. Bil. Dergisi, 33(2), 755-763. doi:https://doi.org/10.35234/fumbd.953296

Çifçi, D., \& Meriç, S. (2021). Pomza-Bazlı Adsorbanların Sentetik $\mathrm{Su}$ Numunelerinden Lityum Adsorpsiyonu Verimliliğinin Karşılaştırılması. Fırat Üniversitesi Müh. Bil. Dergisi, $33(1)$,

185-192. doi:https://doi.org/10.35234/fumbd.773840

Dinçer, İ., \& Ezan, M. A. (2020). Tüba Enerji Depolama Teknolojileri Raporu. Ankara: Türkiye Bilimler Akademisi Yayınları.

Ding, N., Chien , S. W., Andy Hor , T. S., Liu, Z., \& Zong, Y. (2014). Key parameters in design of lithium sulfur batteries. Journal of Power Sources(269), 111-116. doi:https://doi.org/10.1016/j.jpowsour.2014.07.008

Dong, L., Wang, J., Chen, C., Li, H., Zheng, H., Yan, W., . . . Zhang, J. (2021). Acid-treated multi-walled carbon nanotubes as additives for negative active materials to improve highrate-partial-state-of-charge cycle-life of lead-acid batteries. Royal Society of Chemistry, 11, 15273-15283. doi:https://doi.org/10.1039/D1RA02208C

Evers, S., \& Nazar, L. F. (2012). Graphene-enveloped sulfur in a one pot reaction: a cathode with good coulombic efficiency and high practical sulfur content. Chem. Commun(48), 12331235. doi:https://doi.org/10.1039/D1RA02208C

Gül, E., Kılınç, S., \& Gören, A. (2012). Otomatik Kontrol Ulusal Toplantıs1. TOK-2012. Niğde.

Güven, E. C., \& Gedik, K. (2019). Ömrünü Tamamlamış Elektrikli Araç Bataryalarının Çevresel Yönetimi. Journal of the Institute of Science and Technology, 9(2), 726-737. doi:https://doi.org/10.21597/jist.446170

He, H., Ji, X., \& Nazar, L. (2011). High ' C' ' rate Li-S cathodes: sulfur imbibed bimodal porous carbons. Energy Environ. Sci.(4), $2878-2883$ doi:https://doi.org/10.1039/C1EE01219C

Hofmanna, A., Fronczeka, D., \& Besslerc, W. (2014). Mechanistic modeling of polysulfide shuttle and capacity loss in lithium-sulfur batteries. Preprint submitted to Journal of Power Sources, 1-16.

Islam, M., Khalekuzzaman, M., Kabir , S., \& Rana, M. (2021). A Study on Recycling Used Lead-Acid Batteries (ULABs) in Bangladesh. Researchgate. Proceedings of the WasteSafe 2021 - 7th International Conference on Integrated Solid Waste \& Faecal Sludge Management in South-Asian Countries. Khulna, Bangladesh.

Ji, X., Lee, K., \& Nazar, L. (2009). A highly ordered nanostructured carbon-sulphur cathode for lithium-sulphur batteries. $\quad$ Nature $\quad$ Materials(8), 500-506. doi:http://www.nature.com/doifinder/10.1038/nmat2460

Jin, J., Wen, Z., Ma, G., Lu, Y., Cui, Y., Wu, M., . . . Wu, X. (2013). Flexible self-supporting graphene-sulfur paper for lithium sulfur batteries. RSC Adv.(3), 2558-2560. doi:https://doi.org/10.1039/C2RA22808D

Jung, H. Y., Kim, S. C., Shim, J. Y., Mandal, S., Thangarasu , S., \& Thong , T. P. (2021). Positive electrode active material development opportunities through carbon addition in the lead-acid batteries: A recent progress. Journal of Power Sources, 485(229336). doi:https://doi.org/10.1016/j.jpowsour.2020.229336

Kotkunde , N., Amrith Ashwin, K. V., Gosavi , A., \& Gaur, M. (2021). Life Cycle Assessment Of Nickel Cadmium Battery. IOP Conf. Ser.: Mater. Sci. Eng., 11 pages. doi:http://dx.doi.org/10.1088/1757-899X/1123/1/012022

Kozak, M., \& Kozak, Ş. (2012). Enerji Depolama Yöntemleri. SDU International Technologic Science, 4(2), 17-29.

Kul, B. (2020). Geçmişten Günümüze Piller. Takvim-i Vekayi, 8(1), 104-115.

Kumar, R., Sahoo, S., Joanni, E., Singh, R. K., Tan, W. K., Kar, K. K., \& Matsuda, A. (2019). Recent progress in the synthesis of graphene and derived materials for next generation electrodes of high performance lithium ion batteries. Progress in Energy and Combustion Science, 75(100786), 56 pages. doi:http://dx.doi.org/10.1016/j.pecs.2019.100786

Kurzweil, P. (2010). Gaston Planté and his invention of the leadacid battery-The gen- esis of the first practical rechargeable battery. J. Power Sources, 195(14), 4424-4434. doi:https://doi.org/10.1016/j.jpowsour.2009.12.126

Küçükdeveci, N. (2018). Şarj Edilebilir Nikel-Metal Hidrür (Ni$\mathrm{MH}$ ) Pillerinde Kullanılan Hidrojen Depolama Alaşımlarındaki SonGelişmeler. BEÜ Fen Bilimleri Dergisi, 454-472.

Lach , J., Wróbel , K., Wróbel , J., \& Czerwin' ski , A. (2021). Applications of Carbon in Rechargeable Electrochemical Power Sources: A Review. A Review. Energies, 14(2649), 29 pages.

Lin , X., Khosravinia, K., Hu, X., Li , J., \& Lu , W. (2021). Lithium Plating Mechanism, Detection, and Mitigation in Lithium-Ion Batteries. Progress in Energy and Combustion Science, $\quad 87(100953), \quad 30 \quad$ pages. doi:https://doi.org/10.1016/j.pecs.2021.100953

Liu, Y., \& Cui, Y. (2017). Lithium Metal Anodes: A Recipe for Protection. Joule(20), 643-650. doi:https://doi.org/10.1016/j.joule.2017.12.001

Lu, X., Luo, F., Tian, Q., Zhang, W., Sui, Z., \& Chen , J. (2021). Anatase TiO2 nanowires intertangled with CNT for conductive additive-free lithium-ion battery anodes. Journal of Physics and Chemistry of Solids, 153(110037), 7 pages. doi:https://doi.org/10.1016/j.jpcs.2021.110037

Lyu, Y., Wu, X., Wang, K., Feng, Z., Cheng, T., Liu, Y., .. . Guo, B. (2021). An Overview on the Advances of LiCoO2 Cathodes for Lithium-Ion Batteries. Adv. Energy Mater., 11,1-29. doi:https://doi.org/10.1002/aenm.202000982

Ma, Z., Huang, X., Jiang, Q., Huo, J., \& Wang, S. (2015). Enhanced Cycling Stability of Lithium-Sulfur batteries by Electrostatic-Interaction. Electrochimica Acta(182), 884890. doi:https://doi.org/10.1016/J.ELECTACTA.2015.10.009

Makuza, B., Tian, Q., Guo , X., Chattopadhyay, K., \& Yu , D. (2021). Pyrometallurgical options for recycling spent lithiumion batteries: A comprehensive review. Journal of Power 
Sources, $\quad 491(229622), \quad 21 \quad$ pages. doi:https://doi.org/10.1016/j.jpowsour.2021.229622

Martinez-Bolanos, J. R., Morales Udaeta, M. E., Veiga Gimenes, A. L., \& Oliveira da Silva, V. (2020). Economic feasibility of battery energy storage systems for replacing peak power plants for commercial consumers under energy time of use tariffs. Journal of Energy Storage, 29(101373). doi:https://doi.org/10.1016/j.est.2020.101373

Moller, K. T., Jensen, T. R., Akiba, E., \& Li, H. W. (2017). Hydrogen - A sustainable energy car- rier. J. Prog. Nat. Sci., 27, 34-40. doi:https://doi.org/10.1016/j.pnsc.2016.12.014

Muratoğlu, Y., \& Alkaya, A. (2016). ELEKTRIKLLİ ARAÇ TEKNOLOJISİ ve PİL YÖNETIM SİSTEMİ-İNCELEME. VIII. Yenilenebilir Enerji Kaynakları Sempozyumu bildiriler kitabı (s. 10-14). içinde

Muthu , P., \& Sinnaeruvadi , K. (2021). Reversible kinetics and rapid tunnelling characteristics of silicon doped magnesiumtitanium nanocomposites prepared by mechanical alloying route for nickel-metal hydride batteries. Materials Chemistry and Physics, 274, 125-129. doi:https://doi.org/10.1016/j.matchemphys.2021.125129

Müller, T., \& Friedrich, B. (2006). Development of a recycling process for nickel-metal hydride batteries. Journal of Power Sources, 158, 1498-1509. doi:https://doi.org/10.1016/j.jpowsour.2005.10.046

Nagao, M., Akitoshi, H., \& Tatsumisago, M. (2011). Sulfurcarbon composite electrode for allsolid-state $\mathrm{Li} / \mathrm{S}$ battery with Li2S-P2S5 solid electrolyte. Electrochimica Acta(56), 60556059. doi:http://dx.doi.org/10.1016/j.electacta.2011.04.084

Nagata, H., \& Chikusa, Y. (2014). Transformation of P2S5 into a Solid Electrolyte with Ionic Conductivity at the Positive Composite Electrode of All-Solid-State Lithium-Sulfur Batteries. . Energy Technol.(2), 753 - 756.

Nandiwale, R. (2021). Review of Types of Batteries in Electric Vehicles. International Research Journal of Engineering and Technology (IRJET), 8(1), 79-86.

Nazghelichi, T., Torabi, F., \& Esfahan, V. (2021). Reducing the charging time of a lead-acid cell in the sense of linear stability analysis. Journal of Energy Storage, 36(102369). doi:https://doi.org/10.1016/J.EST.2021.102369

Nitesh , K. A., \& Ravichandra . (2021). A Study on Battery Controller Design for the Estimation of State of Charge (SoC) in Battery Management System for Electric Vehicle (EV)/Hybrid EV (HEV). SN Computer Science, 2(197), 1-12. doi:https://doi.org/10.1007/s42979-021-00600-0

Odegbemi, F., Idowu, G. A., \& Adebayo, A. O. (2001). Nickel recovery from spent nickel-metal hydride batteries using LIX-84I-impregnated activated charcoal. Environmental Nanotechnology, Monitoring \& Management, 15(100452). doi:https://doi.org/10.1016/J.ENMM.2021.100452

Ouyang, L., Huang, J., Wang, H., Liu, J., \& Zhu, M. (2017). Progress of hydrogen storage alloys for Ni-MH rechargeable power batteries in electric vehicles: a review. Mater. Chem. Phys., 200, 164-178. doi:https://doi.org/10.1016/j.matchemphys.2017.07.002

Ouyang, L., Huang, J., Wang, H., Liu, J., \& Zhu, M. (2017). Progress of hydrogen storage alloys for Ni-MH rechargeable power batteries in electric vehicles: A review. Mater. Chem. Phys., 200, 164-178.

Özcan, Ö. F., Karadağ, T., Altuğ, M., \& Özgüven, Ö. F. (2021). Elektrikli Araçlarda Kullanılan Pil Kimyasallarının Özellikleri ve Üstün Yönlerinin Kiyaslanması Üzerine Bir Derleme Çalışması. GU J Sci, Part A, 8(2), 276-298.
Putois, F. (1995). Market for nickel—cadmium batteries. Journal of Power Sources, 57, 67-70. doi:https://doi.org/10.1016/0378-7753(95)02243-0

Roth, H. A., Davis, C. L., \& Thomson, R. C. (1997). Modeling solid solution strengthening in nickel alloys. Mater. Transact A., 28, 1329-1335.

Saleh, M. M., Bamsaoud, S. F., \& Barfed, H. M. (2021). Optimization of nitric acid properties for chemical recycling of cadmium from spent Ni-Cd batteries. J. Phys.: Conf. Ser., 10 pages. doi:https://doi.org/10.1088/17426596\%2F1900\%2F1\%2F012018

Samanta , A., \& Chowdhuri, S. (2021). Active Cell Balancing of Lithium-ion Battery Pack Using Dual DC-DC Converter and AuXiliary Lead-acid Battery. Journal of Energy Storage, 33(102109). doi:https://doi.org/10.1016/j.est.2020.102109

Sanguesa, J. A., Torres-Sanz , V., Garrido, P., Martinez, F. J., \& Marquez-Barja, J. M. (2021). A Review on Electric Vehicles: Technologies and Challenges. Challenges. Smart Cities, 4, 372-404. doi:https://doi.org/10.3390/smartcities4010022

Silvestri, , L., Forcina, A., Arcese, G., \& Bella, G. (2020). Recycling technologies of nickelemetal hydride batteries: An LCA based analysis. Journal of Cleaner Production, 273(123083). doi:https://doi.org/10.1016/j.jclepro.2020.123083

Stroe, D., Knap, V., Swierczynski, M., \& Schaltz, E. (2019). Electrochemical Impedance Spectroscopy-Based Electric Circuit Modeling of Lithium-Sulfur Batteries During a Discharging State. Ieee Transactions On Industry Applications(55), doi:https://doi.org/10.1109/TIA.2018.2864160

631-637.

Sylwia, W. (2015). Lithium/Sulfur batteries : development and understanding of the working mechanisms. Université Grenoble Alpes.

Tanibata, N., Tsukasaki, H., Deguchi, M., Mori, S., Hayashi, A., \& Tatsumisago, M. (2017). A novel discharge-charge mechanism of a S-P2S5 composite electrode without electrolytes in all-solid-state Li/S batteries. J. Mater. Chem. A(5),

11224-11228. doi:https://doi.org/10.1039/C7TA01481C

Von Handorf, D. E. (2002). The baghdat battery - myth or reality. Platin and Surface Finishing, 89(5), 84-87.

Walus, Sylwia. Lithium/Sulfur batteries : development and understanding of the working mechanisms. Université Grenoble Alpes, 2015. English. 2015.

Wang, J., Lu, L., Choucair, M., Stride, J., Xu, X., \& Liu, H. (2011). Sulfur-graphene composite for rechargeable lithium batteries. Journal of Power Sources(196), 7030-7034. doi:https://doi.org/10.1016/j.jpowsour.2010.09.106

Wang, W., Xu, G., Zhang, L., Ma, C., Zhao, Y., Zhang, H., . . . Han, S. (2021). Electrochemical features of Ce2Ni7-type

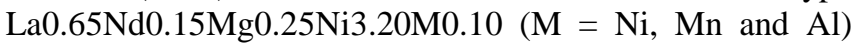
hydrogen storage alloys for rechargeable nickel metal hydride batter. Journal of Alloys and Compounds, 861(158469). doi:https://doi.org/10.1016/j.jallcom.2020.158469

Winter, M., \& Brodd, R. J. (2004). What are batteries, fuel cells, and supercapacitors? Chemical Reviews, 104(10), 42454270. doi:https://doi.org/10.1021/cr020730k

Witantyo, Merdeka, O. P., Amalia, L., Noerochim, L., Setyawan, H., Shahab, A., \& Suwarno, S. (2021). Effects of Graphene Addition on Negative Active Material and Lead Acid Battery performances under Partial State of Charge Condition. Int. J. Electrochem. Sci, 16, $10 \quad$ Pages. doi:http://dx.doi.org/10.20964/2021.08.27 
Wu, D., Shi, F., Zhou, G., Zu, C., Liu, C., Liu, K., . . Cui, Y. (2018). Quantitative investigation of polysulfide adsorption capability of candidate materials for Li-S batteries. Energy Storage Materials(13), 241-246. doi:https://doi.org/10.1016/j.ensm.2018.01.020

Xie , J., \& Lu, Y. C. (2020). A retrospective on lithium-ion batteries. Nature Communications, 11(2499), 4 pages. doi:https://doi.org/10.1038/s41467-020-16259-9

Xu, B., Qian, D., Wang, Z., \& Meng, Y. S. (2012). Recent progress in cathode materials research for advanced lithium ion batteries. Materials Science and Engineering $R$ 73, 5165. doi:https://doi.org/10.1038/s41467-020-16259-9

Xu, J., Dou, S., Liu, H., \& Dai , L. (2013). Cathode materials for next generation lithium ion batteries. Nano Energy, 2, 439442. doi:https://doi.org/10.1016/j.nanoen.2013.05.013

Xu, R., Yue, J., Liu, S., Tu, J., Han, F., Liu, P., \& Wang, C. (2019). Cathode-Supported All-Solid-State Lithium- Sulfur Batteries with High Cell-Level Energy Density. Energy Lett.(4), 1073-1079. doi:https://doi.org/10.1021/acsenergylett.9b00430

Ye, X., Ma, J., Hu, Y., Wei, H., \& Ye, F. (2016). MWCNT porous microspheres with an efficient 3D conductive network for high performance lithium- sulfur batteries. Mater. Chem. A.(4), 775-780. doi:https://doi.org/10.1039/C5TA08991C

Yılmazoğlu, Z. (2010). Isı Enerjisi Depolama Yöntemleri ve Binalarda Uygulanması. Politeknik Dergisi Journal of Polytechnic, 13(1), 33-42.

You, S., Tan, H., Wei, L., Tan, W., \& Li , C. C. (2021). Design Strategies of Si/C Composite Anode for Lithium-Ion Batteries. Chem. Eur. J., 27, 12237 - 12256. doi:https://doi.org/10.1002/chem.202100842

Yuan, Y., Zheng, G., \& Cui, Y. (2013). Nanostructured sulfur cathodes. Chem. Soc.(42), 3018-3032. doi:https://doi.org/10.1039/C2CS35256G

Zhang, Q. (2013). The Current Status on the Recycling of Leadacid Batteries in China. Int. J. Electrochem. Sci., 8, 6457 6466.

Zhang, S. (2013). New insight into liquid electrolyte of rechargeable lithium/sulfur battery. Electrochimica Acta.(97), 226-230. doi:https://doi.org/10.1016/j.electacta.2013.02.122

Zhang, S., \& J.A., R. (2012). A new direction for the performance improvement of rechargeable lithium/sulfur batteries. Journal of Power Sources(200), 77-82. doi:https://doi.org/10.1016/j.jpowsour.2011.10.076

Zhao, M.-Q., Liu, X.-F., Zhang, Q., Tian, G.-L., Huang, J.-Q., Zhu, W., \& Wei, F. (2012). Graphene/Single-Walled Carbon Nanotube Hybrids: One-Step Catalytic Growth and Applications for High-Rate Li-S Batteries. ACS Nano(6), 10759-10769. doi:https://doi.org/10.1021/nn304037d

Zheng, L. K., Wu, K. S., Li, Y., Qi, Z. L., Han, D., Zhang, B., . . . Huo, X. (2008). Blood lead and cadmium levels and relevant factors among children from an e-waste recycling town in China. Environ. Res., 108(15-20). doi:https://doi.org/10.1016/j.envres.2008.04.002

Zhou, Y. Y., Zhang, Z. Y., Zhang, H. Z., Li, Y., \& Weng, Y. (2021). Progress and perspective of vanadium based cathode materials for lithium ion batteries. Tungsten, 3, 279-288. doi:https://doi.org/10.1007/s42864-021-00101-w 